\title{
CASE REPORT: ANKYLOSING SPONDYLITIS AND HIV PATIENT USING ANTI-TNF
}

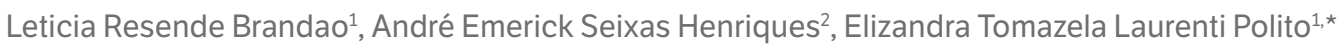 \\ 1.Hospital Estadual Dorio Silva, Serra (ES), Brazil; 2.Multiscan Medicina Diagnóstica, Vitória (ES), Brazil. \\ *Corresponding author: eliz.polito@gmail.com
}

\section{BACKGROUND}

We report a case of a patient with ankylosing spondylitis (AS) using anti-TNF and who was diagnosed with HIV during treatment and maintained the anti-TNF after controlling the viral load.

\section{CASE REPORT}

A 38-year-old male (diagnosed with AS 6 months ago) entered the office in September 2018 with chronic inflammatory low back pain, morning stiffness longer than $1 \mathrm{~h}$, fatigue, HLAB27 present, grade 3 sacroiliitis, and positive response to NSAID. He reports previous episodes of pain in elbows, knees and was using NSAID and methotrexate $20 \mathrm{mg} /$ week. There was improvement in the peripheral condition, but it maintained inflammatory axial pain. Depressed facies. Maintained naproxen and associated amitriptyline $25 \mathrm{mg} /$ day, tramadol. Screening was done to initiate anti-TNF including negative serologies for Hepatitis B, C and HIV, VHS 50 and PCR 49; BASDAI 7, ASDAS-PCR 5.52. Sacroiliac RNM October 2018: chronic sacroiliitis, with focal, active inflammatory process (Figures 1-3). Started golimumab in December 2018, with significant improvement of the clinical picture in the second dose. In March2019, VHS 02 and PCR 0.8, BASDAI 1.2 and ASDAS-PCR inactive. From the second dose on, golimumab was maintained in monotherapy. After 8 months, he lost weight and presented IVAS. After investigation, HIV positivity was evidenced, with high viral load. He underwent joint follow-up with infectology, suspended golimumab and restarted medication for chronic pain with pregabalin and amitriptyline. Started the cocktail for HIV and, after four months, the viral load was zeroed. In effect of the clinically worsening of inflammatory low back pain, BASDAI 6 and, after a shared decision with infectology, we opted for the reintroduction of golimumab. Patient remains asymptomatic, using golimumab in monotherapy and antiretroviral cocktail. Keeping viral load to zero in 2019, 2010 and 2021. Some HIV-infected patients do not develop the full spectrum of clinical manifestations of spondyloarthritis. The predominant rheumatic manifestations exhibited include Achilles tendinitis, low back pain, dactylitis, plantar fasciitis, and shoulder pain. Psoriasiform rashes are common. And a lower frequency of uveitis is observed. In MRI and US, the most common findings include knee synovitis and extensive polyenthesitis.

\section{CONCLUSION}

There are challenges in the management of inflammatory rheumatic diseases in HIV-positive patients, including difficulty in evaluating disease activity and limited information on the safety of immunosuppressive drugs in these individuals. Records for prospective follow-up of HIV patients with arthropathies are urgently needed to clarify the clinical characteristics and natural history of these conditions and develop a treatment protocol.

\section{KEYWORDS}

Ankylosing Spondylitis, HIV, anti-TNF, Golimumabe. 


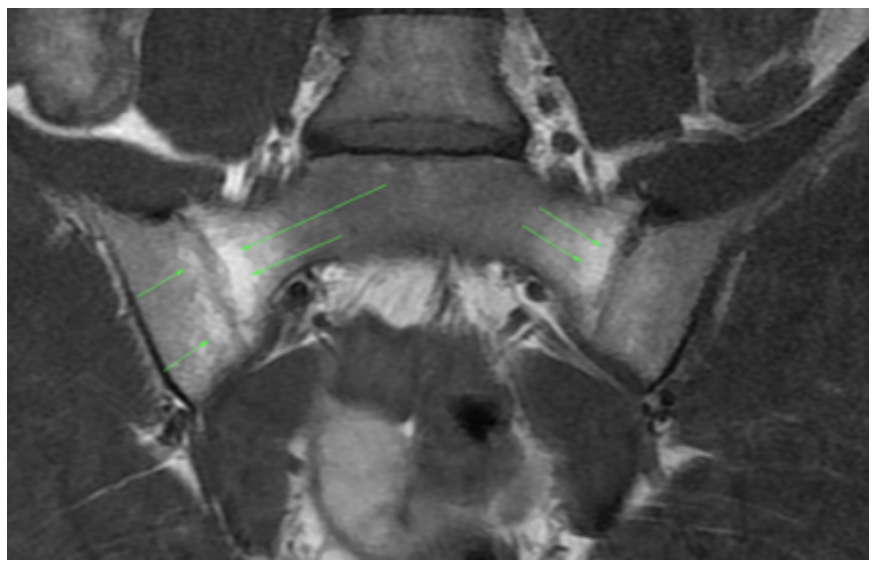

Figure 1. Areas of subchondral liposubstitution due to chronic inflammatory process

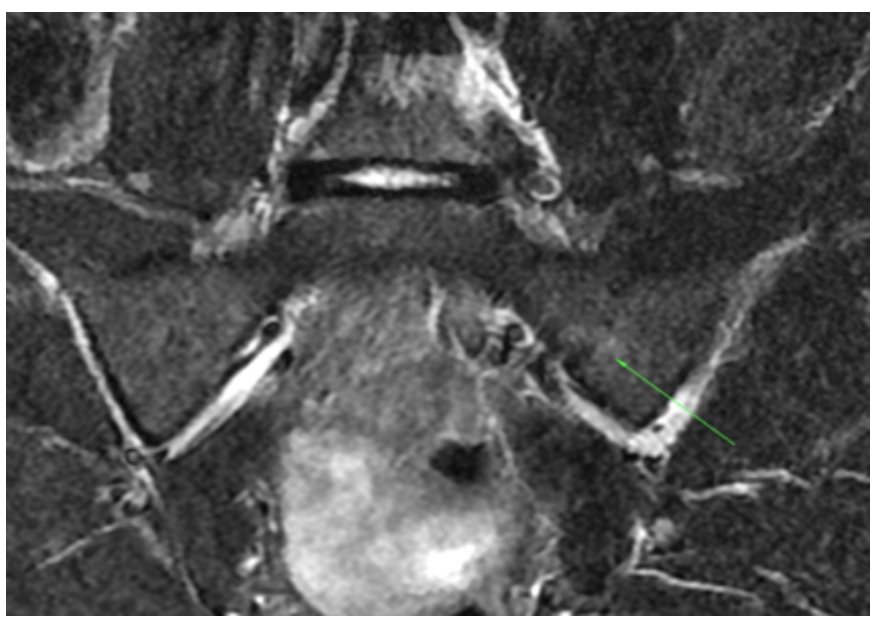

Figure 2. Subchondral edema

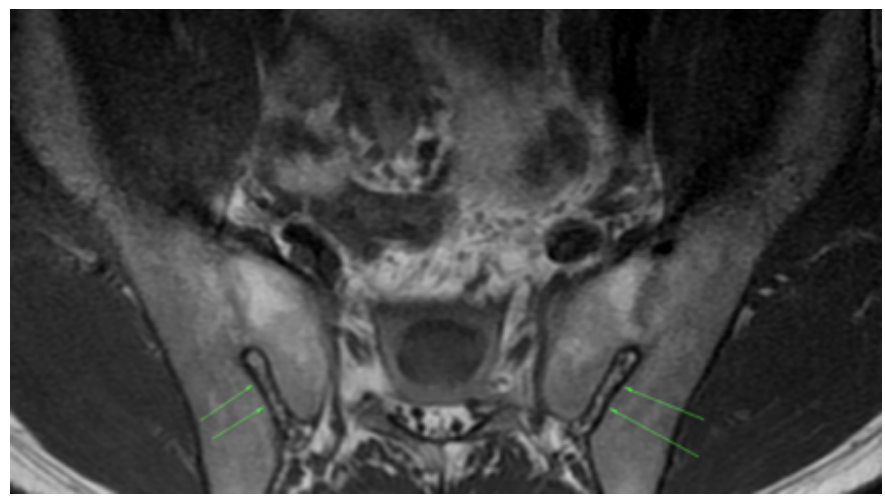

Figure 3. Fibrous portion spared 